\title{
Visual Attention in Driving: The Effects of Cognitive Load and Visual Disruption
}

\author{
Yi-Ching Lee, University of Illinois at Urbana-Champaign, Savoy, Illinois, and John D. Lee \\ and Linda Ng Boyle, University of lowa, lowa City, lowa
}

\begin{abstract}
Objective: This study investigates the effect of cognitive load on guidance of visual attention. Background: Previous studies have shown that cognitive load can undermine driving performance, particularly drivers' ability to detect safety-critical events. Cognitive load combined with the loss of exogenous cues, which can occur when the driver briefly glances away from the roadway, may be particularly detrimental. Method: In each of two experiments, twelve participants engaged in an auditory task while performing a change detection task. A change blindness paradigm was implemented to mask exogenous cues by periodically blanking the screen in a driving simulator while a change occurred. Performance measures included participants' sensitivity to vehicle changes and confidence in detecting them. Results: Cognitive load uniformly diminished participants' sensitivity and confidence, independent of safety relevance or lack of exogenous cues. Periodic blanking, which simulated glances away from the roadway, undermined change detection to a greater degree than did cognitive load; however, drivers' confidence in their ability to detect changes was diminished more by cognitive load than by periodic blanking. Conclusion: Cognitive load and short glances away from the road are additive in their tendency to increase the likelihood of drivers missing safety-critical events. Application: This study demonstrates the need to consider the combined consequence of cognitive load and brief glances away from the road in the design of emerging in-vehicle devices and the need to provide drivers with better feedback regarding these consequences.
\end{abstract}

\section{INTRODUCTION}

Many drivers are transforming their vehicles into mobile offices with devices that allow them to use the Internet, send and receive faxes, receive news, and converse on cell phones (Dewar \& Olson, 2002). These systems promise benefits of increased comfort, productivity, and mobility. However, such devices may also distract drivers and undermine driving safety (Goodman, Tijerina, Bents, \& Wierwille, 1999; J. D. Lee \& Strayer, 2004).

Driving makes intense demands on visual perception (Dewar \& Olson, 2002). As a result, operating devices that require glances away from the road results in structural interference, which can have obvious negative effects on driving performance. Increasing the duration of glances away from the road increases the probability of lane de- parture, such that glances of $2 \mathrm{~s}$ lead to 3.6 times more lane departures than do glances of $1 \mathrm{~s}$ (Green, 1999).

Cognitive interference has less obvious consequences. Operating devices that do not require glances away from the road, such as speech recognition systems, can nevertheless impose a cognitive load that may interfere with driving performance. This cognitive load has the potential to impair drivers' ability to maintain vehicle control (Rakauskas, Gugerty, \& Ward, 2004). Cognitive load can also delay or interrupt cognitive processing of roadway-related information, resulting in longer reaction times (Alm \& Nilsson, 1994, 1995; J. D. Lee, Caven, Haake, \& Brown, 2001), degraded speed and headway control (Strayer \& Drews, 2004), and less effective use of environmental cues to anticipate when to brake (Jamson, Westerman, Hockey, \& Carsten, 2004). 
The effect of cognitive and structural interference depends on the type of task. Multiple resource theory suggests that two tasks that draw upon the same mode (e.g., information received through the eye only or through the eye and the ear), code (i.e., analogue/spatial vs. categorical/verbal processes), or stage of processing (e.g., perceptual, cognitive, the selection and execution of response) will interfere with each other more than two tasks that draw upon different resources (Wickens, 1984, 2002). In driving, a concurrent spatial task interferes with drivers' eye movements to a larger degree than does a concurrent verbal task (Recarte $\&$ Nunes, 2000). Cognitive interference is greatest for tasks that demand the same resources.

Further, recent extensions of multiple resource theory identified separate visual processing resources: ambient and focal. In driving, ambient vision supports lane keeping and focal vision is critical for event detection (Wickens, 1984, 2002). A meta-analysis of the effect of cell phone use on driving performance showed that handheld phones, which demand focal processing, had a relatively small effect on lane keeping but that hands-free phones had a substantial effect on event detection and response (Horrey \& Wickens, 2006). However, even tasks that draw upon different resources, such as cell phone conversations (auditory and verbal) and driving (visual, motor, and spatial), can compete for central processing resources (Pashler, 1998). Such competition can undermine drivers' ability to respond to the roadway environment.

One possible cause of the failure to respond to the environment is that performing a secondary task degrades the encoding and transferring of foveated visual information into short-term memory. Studies have shown that drivers detected (McCarley et al., 2004) and recognized (Strayer, Drews, \& Johnston, 2003) fewer objects when performing a secondary task while driving as compared to driving only; however, the number of fixations on the target region were not different for the two conditions. The difficulty in responding to and recognizing previously fixated stimuli in a dual-task condition may relate to the tendency for one stimulus to interfere with the processing of a subsequent stimulus (Shapiro \& Luck, 1999), such that drivers' attention to a nondriving task interferes with the consolidation of information into short-term memory during fixations.

Failure to respond to the environment may also be caused by disruptions in the distribution of visual attention while performing a secondary task. Several researchers have evaluated eye movement patterns to assess how drivers' inspection behavior changes as a function of cognitive load. Drivers glanced at the mirror and the speedometer less frequently and their distribution of glances to the road became more concentrated when they performed cognitively demanding tasks while driving (Recarte \& Nunes, 2000, 2003). This reduction of the area scanned by the driver could decrease the probability of detecting traffic events because attention is not directed toward those events. Nondriving secondary tasks may disrupt drivers' attention to the roadway, resulting in fewer objects and changes being fixated and attended.

Visual attention can be guided to objects in the visual field by endogenous control (also called goal-driven, conceptually driven, or top-down control) and by exogenous control (also called data-driven, stimulus-driven, or bottom-up control). Endogenous control refers to strategic information processing that occurs when an observer intentionally directs attention toward relevant stimuli. Exogenous control refers to the direction of attention elicited by characteristics of the visual field and implies automatic or mandatory information processing (Jonides, 1981; Posner, 1980; Theeuwes, 1991a).

Previous studies using a cue-target paradigm have manipulated the predictive validity of a centrally located symbolic cue that pointed to one of several stimulus positions to assess the role of endogenous control. These studies have also assessed the role of exogenous control through a nonpredictive abrupt onset (Jonides, 1981; Posner, 1980). Results have generally shown that reaction times are shorter when a target appears in a cued, rather than an uncued, location. Jonides (1981) found an interactive effect such that endogenous control in response to a central predictive cue was affected by concurrent memory load, whereas exogenous control in response to a nonpredictive cue was not.

Generalizing to driving, when information is relevant to the driver, endogenous control purposely directs attention to particular features in the driving environment (Theeuwes, 1991b). On the other hand, exogenous cues, such as abrupt movements, draw attention to a particular object or location without drivers' intention. Based on Jonides's (1981) findings, cognitive load would be expected to interfere more with drivers' attention to 
safety-relevant objects, which is governed by endogenous control, than with their attention to salient objects, which depends on exogenous control.

The change blindness paradigm offers a promising way to assess the effect of cognitive load on visual attention. When changes occur during a brief occlusion of the scene, as in the flicker paradigm, observers have trouble detecting them even when the changes are large, are presented repeatedly, and are expected to occur (Rensink, O'Regan, \& Clark, 1997). Observers do not have trouble detecting changes without the brief occlusion. A common explanation for these findings is that the brief occlusion of the scene disrupts and masks the exogenous cues associated with the abrupt onsets that would normally guide attention to the change (Rensink et al., 1997).

Several variations of the change blindness paradigm support this explanation. When an abrupt onset was added to the prechange image prior to the disruption of the scene, detection was easier if the changed item was the abrupt onset (Scholl, 2000). Likewise, when high-contrast patterns and changes were both presented in a scene, as in the mud splash paradigm, observers struggled to detect changes because the high-contrast patterns served as exogenous cues that drew attention away from the location of the change (O'Regan, Rensink, \& Clark, 1999). Such results suggest that the brief disruption in the change blindness paradigm interferes with change detection by masking abrupt onsets that normally support exogenous control of visual attention (Jonides, 1981; Simons \& Rensink, 2005).

Consistent with the characteristics of endogenous control, in the presence of a brief disruption, objects that are more meaningful (Pringle, Irwin, Kramer, \& Atchley, 2001), are more relevant to traffic safety (Dornhoefer, Unema, \& Velichkovsky, 2002), or are of central interest (e.g., objects considered to be important in the scene; Rensink et al., 1997) are better detected. Others have observed that change detection was impaired when the advantage for changes of central interest was eliminated by inverting the scenes (Kelley, Chun, $\&$ Chua, 2003; Shore \& Klein, 2000).

These results suggest that the change blindness paradigm undermines exogenous control of attention but leaves endogenous control relatively unaffected. It is also possible, however, that in the presence of a brief disruption, observers must rely on visual short-term memory to determine if there is a change (Hollingworth \& Henderson, 2002; Hollingworth, Williams, \& Henderson, 2001). Without the brief disruption, memory is less critical in detecting changes because all the information is available to the observer.

In the driving domain, several researchers have used the change blindness flicker paradigm (Rensink et al., 1997) to study how drivers detect roadway events. According to this paradigm, participants view a sequence of unaltered and altered images of a traffic scene from the driver's perspective, with a brief gray screen between the images (McCarley et al., 2004; Richard et al., 2002). Cognitive load undermined detection of driving-relevant (objects that contained important driving information) and driving-irrelevant (details that were not associated with driving) changes to a similar degree (Richard et al., 2002). In another study, there was a tendency for cognitive load to impair knowledge-driven orienting of attention in older adults but not in younger drivers (McCarley et al., 2004).

In related work, Zheng (2004) developed a dynamic change blindness paradigm in which he asked drivers to detect changes that occurred during brief disruptions in a simulator drive. The results indicated that detection of safety-relevant changes (vehicles that changed location in traffic lanes) was more affected by cognitive load than was detection of safety-irrelevant ones (changes in vehicle features in traffic lanes). However, safety relevance was confounded with vehicle location and vehicle features, making a definitive interpretation of these data difficult. Generally speaking, cognitive load undermines detection of changes that are relevant to the driving task more than it undermines detection of irrelevant changes. These results have partially confirmed Jonides's (1981) finding that cognitive load was particularly detrimental to the endogenous control of attention.

Previous studies have not, however, addressed the combination of cognitive load with and without visual disruption in a dynamic driving environment. Whether short glances away from the driving scene and cognitive load have an additive or interactive effect on drivers' ability to detect changes has important practical and theoretical implications.

The objective of the current study is to compare the effects of cognitive load on the endogenous and exogenous guidance of visual attention by 
using a dynamic change blindness paradigm. Changes that occurred during driving scenarios were masked by a 1-s gray screen so that the effects of cognitive load on endogenous and exogenous control of attention could be compared. The duration of the visual disruption simulated the time drivers might glance away from the forward view to either check the rearview mirror or interact with an in-vehicle information system (Sodhi, Reimer, \& Llamazares, 2002). It was anticipated that cognitive load would diminish endogenous, rather than exogenous, control. Specifically, cognitive load was expected to undermine change detection to a greater degree in the presence of visual disruptions than in the absence of visual disruptions.

\section{EXPERIMENT 1: CHANGE DETECTION AND SAFETY RELEVANCE}

Adynamic change blindness paradigm (Zheng, 2004) was implemented in a driving simulator. A brief visual disruption was designed to remove the transients that normally accompany changes in the visual field, leaving visual attention to be guided by endogenous control. An in-vehicle information system imposed a cognitive loading task that required drivers to listen to auditory messages and respond to questions.

\section{Method}

Participants. Twelve native English speakers (5 men and 7 women) participated in the experiment. Participants ranged in age from 22 to 28 years, with an average age of 25 years $(S D=2.2)$. All drivers were screened for visual acuity, color perception, and depth perception using an Optec Vision Tester. The drivers had at least 5 years of driving experience, drove at least three times per week, and possessed a valid driver's license. Participants were paid $\$ 15$ per hour, with additional compensation (up to $\$ 10$ ) based on auditory task performance. The purpose of providing a bonus was to encourage participants to engage in the secondary task.

Apparatus and tasks. A fixed-based, mediumfidelity driving simulator was used to conduct the experiment. The simulator uses a 1992 Mercury Sable vehicle cab that has been modified to include a $50^{\circ}$ visual field of view, force feedback steering wheel, and a rich audio environment. The fully textured graphics are generated by DriveSafety's Vection $^{\mathrm{TM}}$ software, which delivers a $60-\mathrm{Hz}$ frame rate at $1024 \times 768$ resolution. Data were collected at a rate of $60 \mathrm{~Hz}$.

Each of the four driving scenarios included a straight, four-lane suburban road with a parking lane on each side. Each drive was approximately 6.5 miles $(10.4 \mathrm{~km})$ long, and participants were asked to maintain a speed of $30 \mathrm{mph}(48.3 \mathrm{~km} / \mathrm{hr})$. The drive took approximately $13 \mathrm{~min}$ to complete. Participants were instructed to drive normally, as they would in a real driving environment.

During two of the four drives, the change detection task was administered using a dynamic change blindness paradigm. The projection screen was blanked for $1 \mathrm{~s}$ and replaced with a homogeneous gray screen. Participants were told that the projection screen might blank and that a change to one of the surrounding vehicles could occur during the blank. In the other two drives, participants were told that the screen would not blank but that changes would occur during the drive.

Changes occurred when participants reached predesignated locations. These predesignated locations were situated approximately every $200 \mathrm{~m}$, or every $15 \mathrm{~s}$ if the driver maintained the recommended speed. Participants were asked to identify the type of change by pressing buttons on the steering wheel. Two response buttons on the left of the steering wheel were used to identify forward and backward vehicle changes in the traffic lane. Two response buttons on the right were used to identify movement changes (forward and backward) and property changes (color and identity) in the parking lane. Buttons were labeled so participants could easily identify which to use.

While driving, participants were also asked to listen to and respond to an auditory task (Reyes \& Lee, 2004), which presented information about cost (one or two dollar signs), quality (one or two stars), and wait time (short or long) at three different restaurants. The following is an example of an auditory message: "There are three restaurants located in the area. One restaurant is Louee's Diner, which has an average entrée price of one dollar sign and a quality rating of one star. There is currently a long wait time at Louee's Diner. Another restaurant is Pat's Place, which has an average entrée price of one dollar sign and a quality rating of two stars. There is currently a long wait time at Pat's Place. The last restaurant is Tee Jay's Pizza, which has an average entrée price of two dollar signs and a quality rating of two stars. There is currently a short wait time at Tee Jay's Pizza." 
Questions posed at the end of each message required participants to transform the presented information and relate it to categories of restaurants. For example, a question, delivered in an auditory format, was "Which restaurant could have an average entrée price of $\$ 5$ and has a quality rating of more than 10 positive recommendations?" Participants learned the definitions of the restaurant categories and were given two sample messages during the practice session. They were required to answer each question verbally with the appropriate restaurant name and were encouraged to provide their best answer if they were unsure. The voice of the auditory stimuli was a synthetic English-speaking male adult.

Experimental design and independent variables. The experiment was a 2 (blanking: blank, no blank) $\times 3$ (change: forward, backward, parked vehicle) $\times 2$ (auditory task: task, no task) withinsubjects design. Each participant drove four experimental drives: two contained blanking of the screen (blank) and the other two did not (no blank). The order of the drives was counterbalanced according to a Latin square design. There were three possible changes to the vehicles in front of the participant vehicle, which had different degrees of safety relevance. The backward changes in the traffic lane were considered to be more safety relevant and the forward changes in the traffic lane were considered to be less safety relevant. The changes to parked vehicles were considered to be safety irrelevant.

Both lead and parked vehicles were initially located $60 \mathrm{~m}$ ahead of the participant vehicle. The forward change moved the lead vehicle in the right lane (directly ahead of the participant) forward $18 \mathrm{~m}$. The backward change moved the lead vehicle in the right lane $18 \mathrm{~m}$ closer to the participant vehicle. A parked-vehicle change consisted of either changing the vehicle's location along the parking lane (backward or forward) by $18 \mathrm{~m}$, or changing its color or identity. Each type of change was encountered 12 times during a drive, and each change was accompanied by a screen blanking in the blank condition. Twelve no-change catch trials were included to prevent participants from associating changes with the blanking. The same 36 changes occurred at different locations in the noblank condition.

One drive from each blanking condition contained an auditory task with four unique message sets. Each message was played twice for a total of $150 \mathrm{~s}$. Immediately after the repetition of the message, drivers were asked six questions about the restaurants.

Procedure. After participants signed the necessary Institutional Review Board consent forms, they were introduced to the driving, change detection, and auditory tasks. They then drove a 10-min practice drive to become familiar with the dynamics of the simulator and experience the change detection task and the message system. For each drive, participants were instructed to always maintain their position in the center of the right lane. Drivers were also instructed to press one of the response buttons when they detected a change.

During each auditory task condition, four sets of prerecorded auditory messages were played. Participants were asked to answer the questions as quickly as possible while driving and performing the change detection task. Upon completion of each drive, participants were asked to rate, on a 1 to 10 scale $(1=$ least confident, $10=$ most confi$d e n t)$, their subjective confidence that they had detected the changes and answered the auditory task questions correctly. The experiment took approximately $2 \mathrm{hr}$ to complete.

Dependent variables and scoring. The dependent variables included drivers' sensitivity to changes $\left(d^{\prime}\right)$, confidence ratings, and performance on the auditory task. A signal detection approach was used to analyze change detection performance.

A hit was counted if participants detected a change and correctly pressed the corresponding button within $2.5 \mathrm{~s}$ after the onset of the change event. A miss was counted if, within $2.5 \mathrm{~s}$, participants either failed to press a button or pressed the incorrect button. A false alarm was defined as pressing a button when there was no change. A correct rejection was defined as not pressing any button when there was no change in the blank conditions. In order to count the number of false alarms and correct rejections in the no-blank conditions, we time-stamped 12 predesignated locations to correspond to the 12 no-change catch trials in the blank conditions. The $d^{\prime}$ values were calculated based on the difference between the likelihood of pressing a button correctly when there was a change and the likelihood of pressing a button in the no-change conditions (Macmillan \& Creelman, 2005).

\section{Results}

The effects of the independent variables on $d^{\prime}$ 
and confidence were analyzed with a repeated measures ANOVA. The statistical model was designed to compare the effects of the auditory task and blanking on change detection. Changes were distinguished according to their safety relevance to drivers, with changes that moved toward the drivers being more safety relevant, changes that moved away from drivers being less safety relevant, and changes in the parking lane being safety irrelevant. Results for the color/identity changes in the parking lane were excluded in the analysis because these changes were not comparable to the forward and backward changes in the traffic and parking lanes.

The data were checked to ensure compliance with the normality assumptions (KolmogorovSmirnov test for normality, $p=.058$ ) and homogeneity of variance (Levene's test), for which the $p$ value ranged from .052 to .898 , except for auditory task on confidence, $F(1,142)=5.48, p=.021$. Cohen's $d$ was also calculated to show the magnitude of the effect of the auditory task and blanking on $d^{\prime}$ and confidence. Post hoc tests were conducted using pairwise comparisons with Bonferroni adjustments.

Sensitivity to changes. Participants were less sensitive to vehicle changes during the blank condition, $F(1,121)=34.73, p<.0001, d=0.88$, and the auditory task also diminished sensitivity to changes, $F(1,121)=4.23, p=.042, d=0.28$. The magnitude of the effect of blanking was greater than the effect of the auditory task. The significance of the main effects and nonsignificance of the interaction effect, $F(1,121)=0.50, p=.481$, suggest that blanking and the auditory task had an additive effect on sensitivity (Figure 1).

Participants were most sensitive to changes when the lead vehicle moved backward $\left(d^{\prime}=\right.$ 1.95) toward the participant and least sensitive to parked-vehicle changes $\left(d^{\prime}=1.11\right), F(2,121)=$ $8.98, p=.0002$. The mean sensitivity of forward vehicle changes was 1.61 . The backward movement increased the visual angle of the lead vehicle from $0.86^{\circ}$ to $1.15^{\circ}$, an increase of $33.7 \%$. In contrast, the forward movement decreased the visual angle to $0.67^{\circ}$, a decrease of $22.1 \%$. To determine whether the superior change detection was influenced by size or safety, a subsequent experiment was conducted (Experiment 2).

The interaction between type of change and blanking failed to reach significance, $F(2,121)=$ $2.60, p=.078$, though the means were in the ex-

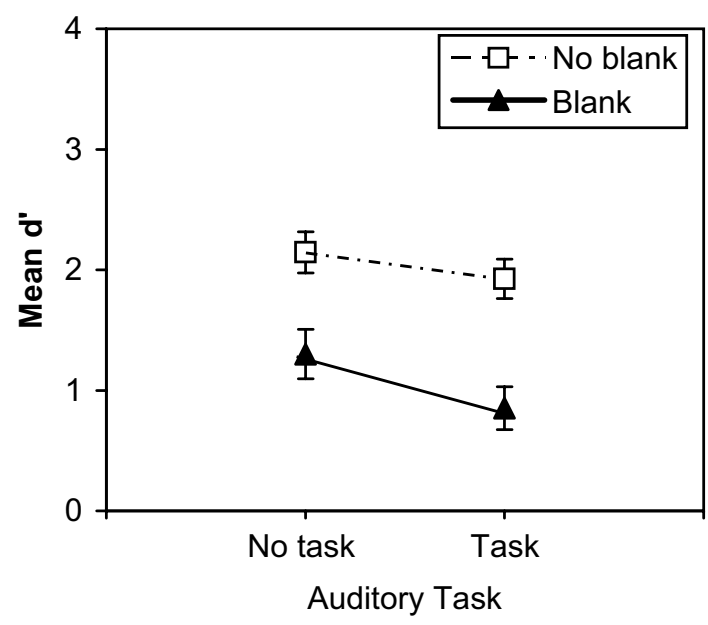

Figure 1. The mean $d^{\prime}( \pm S E)$ as a function of blanking and auditory task in Experiment 1.

pected direction (Figure 2). Parked-vehicle changes were often unnoticed $\left(d^{\prime}=0.38\right)$ when they occurred during blanking. The effect of the auditory task on $d^{\prime}$ was uniform for different types of changes (Figure 2).

Confidence in detecting changes. Participants were less confident in detecting changes during the blank condition, $F(1,121)=9.10, p=.003, d=$ 0.38 , and when they were cognitively loaded with an auditory task, $F(1,121)=19.92, p<.0001, d=$ 0.58 . The magnitude of the effect of the auditory task was greater than that of blanking, which is contrary to the effect sizes for $d^{\prime}$. The interaction between auditory task and blanking was not significant, $F(1,121)=1.46, p=.230$.

Confidence was highest with the backward changes $(M=7.48)$, followed by the forward changes $(M=6.51)$ and, finally, the parked-vehicle changes $(M=5.39), F(2,121)=27.61, p<.0001$. There were no significant interactions between the type of change and either auditory task or blanking.

The relationship between $d^{\prime}$ and confidence was positive in all the experimental conditions. The correlation between $d^{\prime}$ and confidence was significant for the task, $r(72)=.29, p=.014$, and no-task conditions, $r(72)=.55, p<.0001$, and for the blank, $r(72)=.41, p=.0003$, and no-blank conditions, $r(72)=.35, p=.002$.

Secondary task performance. Performance on the auditory task was not strongly related to participants' ability to detect changes, $r(72)=.05, p=$ .666. Participants did not systematically neglect 

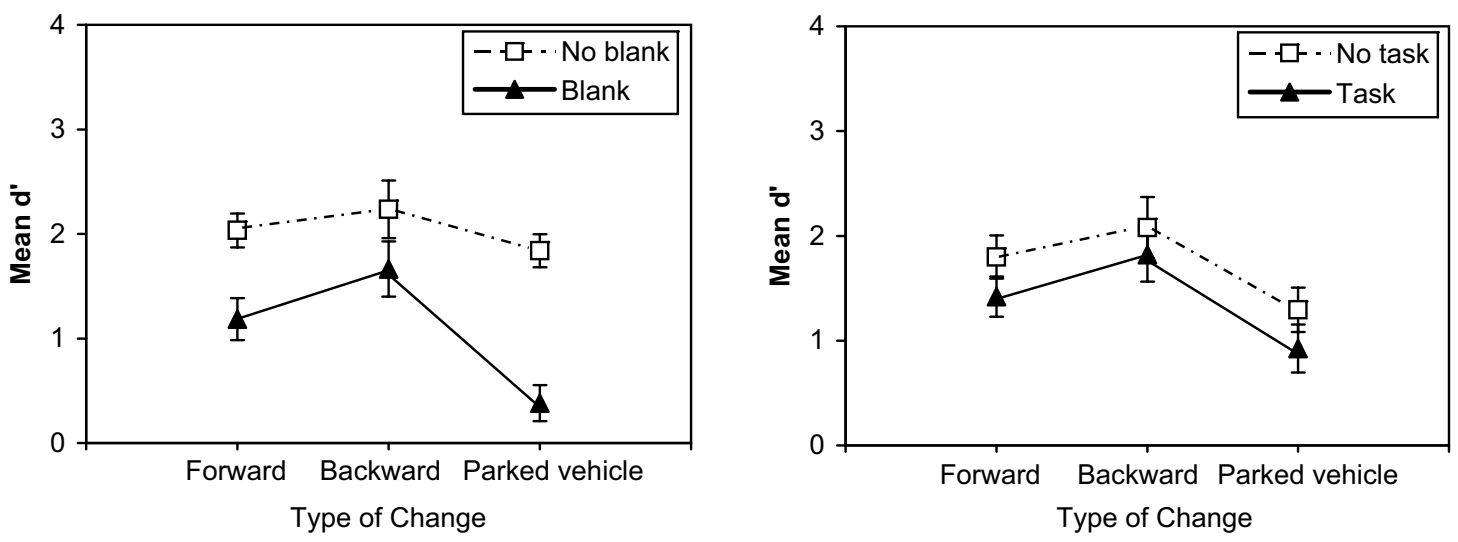

Figure 2. The mean $d^{\prime}( \pm S E)$ as a function of different types of changes and blanking and auditory task in Experiment 1 .

the auditory task to improve their detection performance, nor did they neglect the detection task to focus only on the auditory task. However, participants answered slightly fewer questions correctly during the blank condition $(M=79 \%), F(1,59)=$ $6.42, p=.013$, than during the no-blank condition $(M=83 \%)$. This finding suggests that drivers considered the auditory task secondary to driving and that there was a slight tendency to neglect it when the change detection demands increased.

\section{Discussion}

The introduction of auditory tasks and brief blanking of the driving scene diminished participants' sensitivity to changes as well as their confidence in detecting them. The diminished sensitivity to changes is consistent with Zheng's (2004) findings. Even though the safety-relevant changes were detected more reliably than were the safety-irrelevant changes, cognitive load uniformly diminished the detection of both types of changes. This finding concurs with that of Richard et al. (2002), who observed that performing a nondriving secondary task impaired drivers' ability to detect driving-relevant and driving-irrelevant changes to a similar degree. The decreased confidence in detecting changes suggests that participants were aware that the cognitive load of the auditory task and the blanking both diminished their performance.

Blanking and the auditory task affected $d^{\prime}$ and confidence to different degrees. As compared with the auditory task, blanking had a much stronger effect on drivers' sensitivity to detecting changes; however, the auditory task had a stronger effect on confidence in detecting changes. The stronger effect of blanking on $d^{\prime}$ than on confidence suggests that drivers may not be aware of the influence that brief glances can have on performance. They may think that they detected changes efficiently when in fact they did not. The correlations show a positive relationship between $d^{\prime}$ and confidence, suggesting that participants were aware of the effect of the experimental conditions on their change-detection performance.

We hypothesized that cognitive load would be particularly detrimental to detecting changes during the blanking condition, when endogenous control guides attention. The nonsignificant interactions between auditory task and blanking suggest that cognitive load diminishes detection performance to a similar degree whether or not exogenous cues are available to guide attention. This finding indicates that cognitive load and short visual disruptions are additive in their tendency to undermine detection of roadway events. The lack of an interaction may be the result of drivers compensating by attending less to the auditory task. In fact, participants did answer fewer questions correctly during the blanking condition, in which change detection depended on endogenous control.

Participants were most sensitive to changes when the lead vehicle moved backward. One explanation is that backward movements were more safety relevant and might have required driver intervention. The safety-relevant movement may have influenced the endogenous control of attention, thereby drawing drivers' attention toward it. Another explanation is that this change also caused the image size of the lead vehicle to increase, and 
the retinal expansion may have contributed to a looming cue, making the backward change a salient exogenous cue (D. N. Lee, 1998; Regan \& Vincent, 1995). Experiment 2 was designed to further investigate whether the relatively higher $d^{\prime}$ for backward changes was attributable to the endogenous influence of safety relevance or to the exogenous cue associated with the increased visual angle.

\section{EXPERIMENT 2: IMAGE SIZE AND SAFETY RELEVANCE}

In Experiment 1, participants were most sensitive and confident in detecting backward movements of the lead vehicle. This backward movement made for a larger, more salient exogenous cue. It also imposed a safety-relevant situation and thus made for a stronger endogenous cue as compared with a forward change in the lead vehicle position. Experiment 2 was designed to identify the cause of the higher $d^{\prime}$ for detecting vehicles that moved closer to drivers.

\section{Method}

The protocol for Experiment 2 is discussed only to the extent that it differs from the protocol used in Experiment 1.

Participants. Twelve native English speakers ( 3 men and 9 women) participated in the experiment. Participants ranged in age from 20 to 26 years, with an average age of 22 years $(S D=1.7)$. No participants took part in both experiments.

Apparatus and tasks. Arrangement of the response buttons on the steering wheel was slightly different in Experiment 2: the upper left button corresponded to change-to-left-lane changes, the upper right button corresponded to change-toright-lane changes, the lower left button corresponded to color/identity changes in the parking lane, and the lower right button corresponded to location changes in the parking lane.

Experimental design and independent variables. The experiment used a 2 (blanking: blank vs. no blank) $\times 3$ (change: left vs. right vs. parked vehicle) $\times 2$ (auditory task: task vs. no task) withinsubjects design. A left change moved a vehicle in the right lane to the left lane ahead of the participant vehicle. Aright change moved a vehicle in the left lane to the right lane directly ahead of the participant vehicle. The right changes are of immediate safety relevance to drivers because they place the vehicle directly into the lane in which the participant is driving. In contrast, the left changes are less safety relevant.

The left and right changes were further broken into two location categories: near and far. For both left and right changes, six occurred at the near location and six at the far location. The vehicle arrangements and changes were purposely configured to be comparable to those in Experiment 1. The near location corresponded to the end position of a backward change, and the far location corresponded to the initial position of a backward change. Parked-vehicle changes were the same as those in Experiment 1. A pace car was placed $7 \mathrm{~m}$ ahead of the participant vehicle in the left lane and drove at $30 \mathrm{mph}$. The participants were asked to maintain their speed relative to the pace car and to keep it in sight throughout the drives.

\section{Results}

Results for the color/identity changes in the parking lane were excluded in the analysis. As with the first experiment, the assumptions for normality (Kolmogorov-Smirnov test for normality, $p=.061$ ) and for homogeneity of variance (Levene's test), for which $p$ values ranged from .36 to 1.00 , except for the effect of change type on confidence, $F(2,141)=3.67, p=.028$, were verified before the analysis of variances were conducted.

Sensitivity to changes. Participants were less sensitive to changes during the blank condition, $F(1,121)=44.25, p<.0001, d=0.60$, and while performing the auditory task, $F(1,121)=16.05$, $p=.0001, d=0.35$. As in Experiment 1, the magnitude of the effect of blanking was greater than that of the auditory task. Similar to Experiment 1, the nonsignificant Auditory $\times$ Blanking interaction, $F(1,121)=0.18, p=.674$, suggests that the effects of blanking and cognitive load are additive (Figure 3).

Participants were similarly sensitive to vehicles moving to the left $\left(d^{\prime}=2.79\right)$ or the right $\left(d^{\prime}=\right.$ 2.91) but were less sensitive to the changes to parked vehicles $\left(d^{\prime}=1.15\right), F(2,121)=133.32$, $p<.0001$. To identify the cause of higher $d^{\prime}$ for detecting vehicles that moved closer to drivers, we performed a separate analysis that compared the main effect of change location on $d^{\prime}$. Change location of the moving vehicles affected participants' sensitivity, $F(3,165)=3.90, p=.010$, with greater sensitivity for the close location vehicles $\left(d^{\prime}=2.46\right)$ 


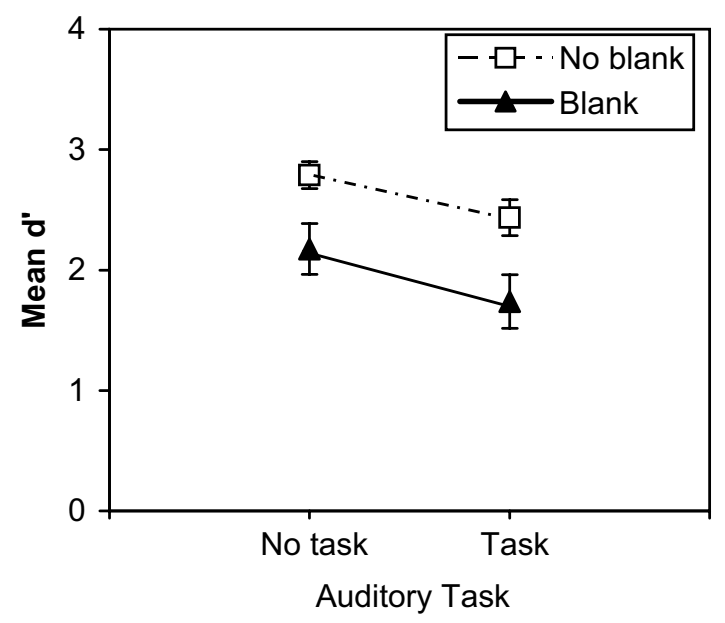

Figure 3. The mean $d^{\prime}( \pm S E)$ as a function of blanking and auditory task in Experiment 2.

compared with the far location vehicles $\left(d^{\prime}=\right.$ 2.25). Post hoc comparisons showed that vehicle changes to the right were detected no better than vehicle changes to the left at close, $t(165)=1.19$, $p=1.000$, and far locations, $t(165)=0.64, p=$ 1.000. This finding suggests that perhaps image size and location, rather than safety relevance alone, affects sensitivity in detecting changes.

The Blanking $\times$ Change Type interaction for $d^{\prime}$ was significant, $F(2,121)=21.39, p<.0001$ (Figure 4). Blanking diminished $d^{\prime}$ for parked-vehicle changes, $t(121)=9.17, p<.0001$, but not for left and right changes. Parked-vehicle changes were often unnoticed $\left(d^{\prime}=0.36\right)$ when they occurred during blanking. The effect of the auditory task on $d^{\prime}$ was uniform for different types of changes

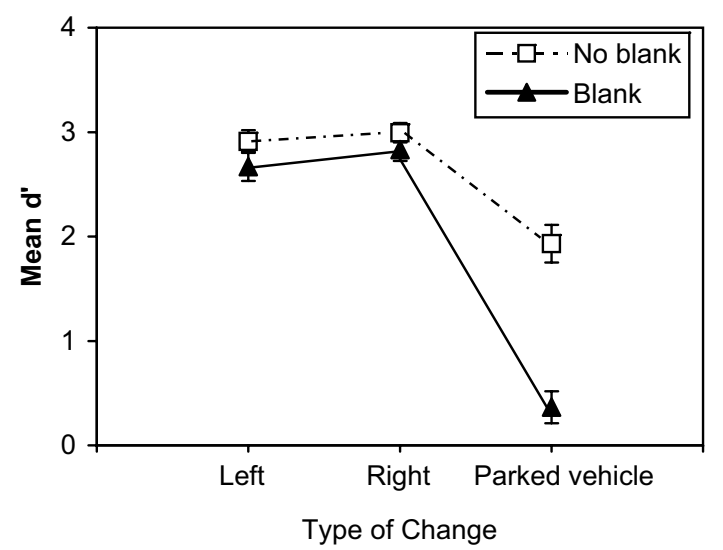

(Figure 4). Neither safety relevance, changes into or out of the drivers' lane, nor centrality of the change affected the degree to which cognitive load impaired detection. Given that blanking had a significant effect on detecting parked-vehicle changes but the auditory task did not, we expected a significant three-way (Auditory Task $\times$ Blanking $\times$ Change Type) interaction. However, our results did not reveal this effect, $F(2,121)=0.38, p=.684$.

Confidence in detecting changes. Consistent with change detection performance, participants were less confident during the blank condition, $F(1,121)=31.53, p<.0001, d=0.50$, and when there was an auditory task, $F(1,121)=29.95, p<$ $.0001, d=0.48$. Unlike Experiment 1, in which cognitive load had a greater effect on confidence than it did on change detection performance, here the effect on confidence was similar to that of sensitivity to change detection, even though blanking had a larger effect on change detection performance.

Participants were similarly confident in detecting the left $(M=7.68)$ and right $(M=7.77)$ changes and were less confident in detecting the parkedvehicle $(M=4.64)$ changes, $F(2,121)=110.71, p<$ .0001 . There were no significant interactions between the type of change and the auditory task or blanking for confidence.

The correlation between $d^{\prime}$ and confidence was significant for the task, $r(72)=.42, p=.0002$, and no-task conditions, $r(72)=.36, p=.001$, and for the blank, $r(72)=.47, p<.0001$, and no-blank conditions, $r(72)=.33, p=.004$. The magnitude of the correlations was comparable between Experiment 2 and Experiment 1.

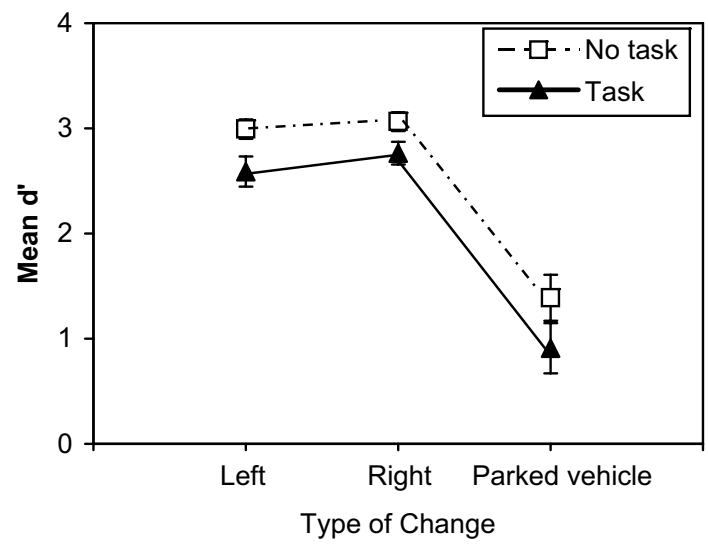

Figure 4. The mean $d^{\prime}( \pm S E)$ as a function of different types of changes and blanking and auditory task in Experiment 2. 
Secondary task performance. There was little evidence of a trade-off between the auditory task and detection performance, $r(72)=.03, p=.772$, suggesting that participants did not neglect the auditory task to improve their detection performance. Contrary to Experiment 1, no differences were observed in secondary task performance between the blank and no-blank conditions, $F(1$, $59)=1.85, p=.179$. It is possible that because the detection task was less demanding with lateral movements, participants could devote more attention to the auditory task.

The magnitude of the effect of the auditory task for the two experiments was similar for $d^{\prime}(0.28$ vs. 0.35$)$ and confidence ( 0.60 vs. 0.50$)$. In contrast, sensitivity in detecting a change in the lead vehicle was substantially lower in Experiment 1 $\left(d^{\prime}=1.78\right)$ than in Experiment $2\left(d^{\prime}=2.85\right)$. It is most likely that the lack of difference in secondary task performance in Experiment 2 was attributable to the more demanding detection task in Experiment 1 .

\section{Discussion}

Consistent with the findings in Experiment 1, the presence of an auditory task diminished participants' sensitivity to changes and their confidence in detecting them. Cognitive load uniformly decreased the detection of all types of changes. The decreased confidence in detecting changes suggests that participants were aware of their performance degradation when they were cognitively loaded with an auditory task. The tendency for cognitive load and short glances to be additive in affecting drivers' sensitivity to changes and confidence in detecting them suggests that drivers will be least sensitive to roadway events when structural and cognitive interference occur simultaneously.

Participants were similar in their sensitivity and confidence in detecting right and left changes, even though the vehicle moving from the left to right lane was assumed to be more safety relevant. Similar to Zheng's (2004) results, drivers were slightly more sensitive to changes at near locations as compared with those at far locations, but they were much less sensitive to changes in the parking lane. In combination, these results suggest that drivers are sensitive to safety-relevant locations, such as the traffic ahead of them, rather than to safety-relevant events. More thorough manipulations of location and safety relevance are needed to confirm these results.
The significant interaction between blanking and change type for $d^{\prime}$ suggests that when searching is guided by endogenous control, changes that are safety irrelevant are less likely to be noticed. However, the concept of safety relevance coincides with spatial location in the current study. We did not have safety-relevant events in the parking lane. Therefore our results could also be explained in terms of the location in the visual field such that drivers pay more attention to objects in the traffic lanes and neglect objects on the side of the road. Our results suggest that exogenous cues may be particularly important in guiding drivers' attention to events that occur away from the center of the road. More research is needed to understand whether drivers attend to objects according to their safety relevance or spatial location.

As in Experiment 1, $d^{\prime}$ was positively related to confidence, suggesting that drivers were aware of how the experimental conditions affected their detection performance. The strength of this relationship was similar in the two experiments, even though the lateral movements of the lead vehicle were more easily detected in the second experiment. Drivers seem to be able to adjust their assessment of their performance on the detection task according to its difficulty.

\section{GENERAL DISCUSSION}

Using a dynamic change blindness paradigm, we designed two experiments to investigate the effect of cognitive load on drivers' ability to detect changes in the driving environment. The dynamic change blindness paradigm provides a situation in which exogenous cues are masked by visual disruptions, resulting in a situation in which visual attention is primarily guided by endogenous control. We hypothesized that cognitive load would diminish drivers' sensitivity and confidence in detecting changes under these circumstances. The results indicate that cognitive load uniformly diminishes participants' sensitivity to changes and their confidence in detecting them, independent of safety relevance or lack of exogenous cues.

Jonides (1981) found that endogenous control was affected by concurrent memory load, whereas exogenous control was not. In his experiment, the demands of a memory task interfered with endogenous control associated with the central cue but left the exogenous control associated with the peripheral cue relatively unaffected. Instead of 
confirming this interactive effect, we found that cognitive load undermined change detection to a similar degree when exogenous cues were masked and when they were not.

In addition, cognitive load undermined detection of safety-relevant and safety-irrelevant events similarly. Therefore, our results suggest that cognitive load undermines both endogenous and exogenous control of attention; the safety relevance or saliency of an object does not guarantee detection if drivers are cognitively loaded. The findings of this study support multiple resource theory in that cognitive load indeed interferes with event detection, both of which require focal processing (Horrey \& Wickens, 2006).

Both experiments also showed that masking exogenous cues greatly diminishes drivers' detection of events that occur away from the center of the roadway. Driver training and experience may lead people to monitor the center of the road and to depend on exogenous cues for safety-relevant events that occur on the side of the road. Such expectations enabled drivers to accommodate the lack of exogenous cues in detecting changes in the center of the road but left them vulnerable to those occurring on the side. Such a process may be an effective adaptation to routine driving situations in which drivers and pedestrians obey the rules of the road, but it may fail when the unexpected occurs. Overall, drivers' ability to detect roadway events is affected by a combination of structural and cognitive interference, with structural interference being particularly detrimental to events away from the center of the road.

An alternative explanation of these results is that cognitive load diminishes event detection primarily because it degrades information consolidation. Drivers miss detecting objects because these objects (even though previously fixated) are not properly consolidated and transferred into short-term visual memory. This is similar to the attentional blink phenomenon (Shapiro \& Luck, 1999), in which drivers may fail to respond appropriately, even if they have looked at objects in the scene, because they do not form a durable shortterm memory of them.

Additional research is needed to understand how drivers scan the environment under cognitive load. Eye movement analyses can provide more insight on how cognitive load influences the way drivers detect objects in the roadway. One possibility is that the probability of fixating certain ob- jects declines when drivers are cognitively loaded. Another possibility is that the probability of detecting a change upon fixation declines. The first alternative would suggest a failing of visual attention, and the second would support a failing of consolidation.

Further, the visual disruptions may not have neatly separated the two mechanisms that guide attention. Unlike many change blindness experiments that present people with unique changes, this experiment included a limited number of changes and locations. In the absence of blanking, the repetition of changes likely led participants to monitor changes according to an attentional set, which suggests that exogenous-guided attention may be influenced by the endogenous factors (Folk, Remington, \& Johnston, 1992).

Furthermore, in situations in which exogenous cues were supposedly eliminated by the visual disruptions, the postblank vehicle that had undergone a backward movement was substantially larger, and the retinal expansion or looming effect (D. N. Lee, 1998; Regan \& Vincent, 1995) may have made the vehicle more salient (Franconeri \& Simons, 2003). Thus, the looming vehicle may provide an additional exogenous cue that is not eliminated by the visual disruptions. The dynamic change blindness paradigm offers a promising, but imperfect, method for assessing the role of endogenous and exogenous control of attention in driving.

The dynamic change blindness paradigm is a more ecologically valid approach to studying how drivers attend to events in the environment, as compared with the static change blindness paradigm. However, its validity is challenged by the artificial technique used to simulate glances away from the road and eliminate exogenous cues associated with roadway changes. In contrast to a natural driving situation, here drivers did not choose when they would "glance away" from the road. In reality, drivers might carefully time glances and be particularly attentive to the situation before and after such a glance. The decrement in change detection observed in this study may overestimate the consequence of short glances away from the road.

In addition, this study required drivers to engage in a cognitively demanding task, one that many drivers might not attempt. However, the number of drivers who use cell phones and even read newspapers while driving suggests that such 
tasks are not beyond what many drivers might attempt in the coming years (Glassbrenner, 2005).

Although the artificial nature of some aspects of this study limit its generalization to actual driving situations, the results show that both cognitive and structural distractions can have profound consequences for detecting changes in the driving environment and that drivers may not always be aware of these consequences. Even brief glances away from the road may make drivers vulnerable to neglecting changes, particularly those occurring in the periphery. This could exacerbate drivers' tendency to neglect safety-critical events that occur to the side of the roadway (Fisher et al., 2002). Drivers' appreciation for these consequences is imperfect. Drivers may underestimate the consequence of seemingly inconsequential distractions - a brief glance - as compared with more obvious distractions. These results suggest that drivers may benefit from feedback regarding how in-vehicle information systems undermine visual attention (Donmez, Boyle, \& Lee, 2003).

\section{ACKNOWLEDGMENTS}

The work presented here is part of the Safety Vehicle(s) Using Adaptive Interface Technology (SAVE-IT) program that was sponsored by the U.S. Department of Transportation, National Highway Traffic Safety Administration (project manager: Michael Perel) and administered by the John A. Volpe National Transportation Systems Center (project manager: Mary D. Stearns). We are also grateful for the contributions of the University of Iowa Public Policy Center faculty and staff for assistance in preparing this manuscript.

\section{REFERENCES}

Alm, H., \& Nilsson, L. (1994). Changes in driver behavior as a function of handsfree mobile phones - A simulator study. Accident Analysis and Prevention, 26, 441-451.

Alm, H., \& Nilsson, L. (1995). The effects of a mobile telephone task on driver behavior in a car following situation. Accident Analysis and Prevention, 27, 707-715.

Dewar, R. E., \& Olson, P. L. (2002). Human factors in traffic safety. Tucson, AZ: Lawyers \& Judges.

Donmez, B., Boyle, L. N., \& Lee, J. D. (2003). Taxonomy of mitigation strategies for driver distraction. In Proceedings of the Human Factors and Ergonomics Society 47th Annual Meeting (pp. 1865-1869). Santa Monica, CA: Human Factors and Ergonomics Society.

Dornhoefer, S. M., Unema, P. J. A., \& Velichkovsky, B. M. (2002). Blinks, blanks and saccades: How blind we really are for relevant visual events. In J. Hyönä, D. P. Munoz, W. Heide, \& R. Radach (Eds.), The brain's eye: Neurobiological and clinical aspects of oculomotor research (pp. 119-131). Amsterdam: Elsevier Science Publishing Co.
Fisher, D. L., Laurie, N. E., Glaser, R., Connerney, K., Pollatsek, A., Duffy, S. A., et al. (2002). Use of a fixed-base driving simulator to evaluate the effects of experience and PC-based risk awareness training on drivers' decisions. Human Factors, 44, 287-302.

Folk, C. L., Remington, R. W., \& Johnston, J. C. (1992). Involuntary covert orienting is contingent on attentional control settings. Journal of Experimental Psychology - Human Perception and Performance, 18, 1030-1044.

Franconeri, S. L., \& Simons, D. J. (2003). Moving and looming stimuli capture attention. Perception and Psychophysics, 65, 999-1010.

Glassbrenner, D. (2005). Driver cell phone use in 2005 - Overall results. Retrieved January 10, 2006, from http://www-nrd.nhtsa. dot.gov/pdf/nrd-30/NCSA/RNotes/2005/809967.pdf

Goodman, M. J., Tijerina, L., Bents, F. D., \& Wierwille, W. W. (1999). Using cellular telephones in vehicles: Safe or unsafe? Transportation Human Factors, 1, 3-42.

Green, P. (1999). Visual and task demands of driver information systems (No. UMTRI-98-16). Ann Arbor: University of Michigan Transportation Research Institute.

Hollingworth, A., \& Henderson, J. M. (2002). Accurate visual memory for previously attended objects in natural scenes. Journal of Experimental Psychology - Human Perception and Performance, 28, 113-136.

Hollingworth, A., Williams, C. C., \& Henderson, J. M. (2001). To see and remember: Visually specific information is retained in memory from previously attended objects in natural scenes. Psychonomic Bulletin and Review, 8, 761-768.

Horrey, W. J., \& Wickens, C. D. (2006). Examining the impact of cell phone conversations on driving using meta-analytic techniques. Human Factors, 48, 196-205.

Jamson, A. H., Westerman, S. J., Hockey, G. R. J., \& Carsten, O. M. J. (2004). Speech-based E-mail and driver behavior: Effects of an in-vehicle message system interface. Human Factors, 46, 625-639.

Jonides, J. (1981). Voluntary versus automatic control over the mind's eye's movement. In J. B. Long \& A. D. Baddeley (Eds.), Attention and performance IX (pp. 187-203). Hillsdale, NJ: Erlbaum.

Kelley, T. A., Chun, M. M., \& Chua, K. P. (2003). Effects of scene inversion on change detection of targets matched for visual salience. Journal of Vision, 3, 1-5.

Lee, D. N. (1998). Guiding movement by coupling taus. Ecological Psychology, 10, 221-250.

Lee, J. D., Caven, B., Haake, S., \& Brown, T. L. (2001). Speech-based interaction with in-vehicle computers: The effect of speech-based E-mail on drivers' attention to the roadway. Human Factors, 43, 631-640.

Lee, J. D., \& Strayer, D. L. (2004). Preface to the special section on driver distraction. Human Factors, 46, 583-586.

Macmillan, N. A., \& Creelman, C. D. (2005). Detection theory: A user's guide (2nd ed.). Mahwah, NJ: Erlbaum.

McCarley, J. S., Vais, M. J., Pringle, H., Kramer, A. F., Irwin, D. E., \& Strayer, D. L. (2004). Conversation disrupts change detection in complex traffic scenes. Human Factors, 46, 424-436.

O’Regan, J. K., Rensink, R. A., \& Clark, J. J. (1999). Change-blindness as a result of "mudsplashes." Nature, 398, 34.

Pashler, H. E. (1998). The psychology of attention. Cambridge, MA: MIT Press.

Posner, M. I. (1980). Orienting of attention. Quarterly Journal of Experimental Psychology, 32, 3-25.

Pringle, H. L., Irwin, D. E., Kramer, A. F., \& Atchley, P. (2001). The role of attentional breadth in perceptual change detection. Psychonomic Bulletin and Review, 8, 89-95.

Rakauskas, M. E., Gugerty, L. J., \& Ward, N. J. (2004). Effects of naturalistic cell phone conversations on driving performance. Journal of Safety Research, 35, 453-464.

Recarte, M. A., \& Nunes, L. M. (2000). Effects of verbal and spatialimagery tasks on eye fixations while driving. Journal of Experiment al Psychology: Applied, 6, 31-43.

Recarte, M. A., \& Nunes, L. M. (2003). Mental workload while driving: Effects on visual search, discrimination, and decision making. Journal of Experimental Psychology: Applied, 9, 119-137.

Regan, D., \& Vincent, A. (1995). Visual processing of looming and time to contact throughout the visual field. Vision Research, 35, $1845-1857$.

Rensink, R. A., O'Regan, J. K., \& Clark, J. J. (1997). To see or not to see: The need for attention to perceive changes in scenes. Psychological Science, 8, 368-373. 
Reyes, M. L., \& Lee, J. D. (2004). The influence of IVIS distractions on tactical and control levels of driving performance. In Proceedings of the Human Factors and Ergonomics Society 48th Annual Meeting (pp. 2369-2373). Santa Monica, CA: Human Factors and Ergonomics Society.

Richard, C. M., Wright, R. D., Ee, C., Prime, S. L., Shimizu, Y., \& Vavrik, J. (2002). Effect of a concurrent auditory task on visual search performance in a driving-related image-flicker task. Human Factors, 44, 108-119.

Scholl, B. J. (2000). Attenuated change blindness for exogenously attended items in a flicker paradigm. Visual Cognition, 7, 377-396.

Shapiro, K. L., \& Luck, S. J. (1999). The attentional blink: A front-end mechanism for fleeting memories. In V. Coltheart (Ed.), Fleeting memories: Cognition of brief visual stimuli (pp. 95-118). Cambridge, MA: MIT Press.

Shore, D. I., \& Klein, R. M. (2000). The effects of scene inversion on change blindness. Journal of General Psychology, 127, 27-43.

Simons, D. J., \& Rensink, R. A. (2005). Change blindness: Past, present, and future. Trends in Cognitive Sciences, 9, 16-20.

Sodhi, M., Reimer, B., \& Llamazares, I. (2002). Glance analysis of driver eye movements to evaluate distraction. Behavior Research Methods, Instruments and Computers, 34, 529-538.

Strayer, D. L., \& Drews, F. A. (2004). Profiles in driver distraction: Effects of cell phone conversations on younger and older drivers. Human Factors, 46, 640-649.

Strayer, D. L., Drews, F. A., \& Johnston, W. A. (2003). Cell phoneinduced failures of visual attention during simulated driving. Journal of Experimental Psychology: Applied, 9, 23-32.

Theeuwes, J. (1991a). Exogenous and endogenous control of attention The effect of visual onsets and offsets. Perception and Psychophys ics, 49, 83-90.

Theeuwes, J. (1991b). Visual selection: Exogenous and endogenous control. In A. G. Gale, I. D. Brown, C. M. Haslegrave, H. W. Kruysse, \& S. P. Taylor (Eds.), Vision in Vehicles III (pp. 53-61). Amsterdam: North-Holland/Elsevier Science.

Wickens, C. D. (1984). Processing resources in attention. In R. Parasuraman \& D. R. Davies (Eds.), Varieties of attention (pp. 63-101). San Diego, CA: Academic Press.
Wickens, C. D. (2002). Multiple resources and performance prediction. Theoretical Issues in Ergonomics Science, 3, 159-177.

Zheng, X. (2004). Exploring the driver's situation awareness in a dynamic traffic environment. Unpublished Ph.D. dissertation, University of Illinois at Urbana-Champaign, Urbana, IL.

Yi-Ching Lee is an assistant professor of human factors within the Institute of Aviation at the University of Illinois at Urbana-Champaign, where she holds appointments in the Beckman Institute for Advanced Science and Technology and the Department of Industrial and Enterprise Systems Engineering. She received her Ph.D. in industrial engineering in 2006 from the University of Iowa.

John D. Lee is a professor of industrial engineering and director of the Cognitive Systems Laboratory at the University of Iowa. He received his Ph.D. in mechanical engineering in 1992 from the University of Illinois at Urbana-Champaign.

Linda Ng Boyle is an assistant professor at the University of Iowa with joint appointments in the Department of Mechanical and Industrial Engineering and Public Policy Center. She received her Ph.D. in civil and environmental engineering in 1998 from the University of Washington.

Date received: September 15, 2005

Date accepted: August 24, 2006 